\title{
Hopf bifurcation of a heroin model with time delay and saturated treatment function
}

\section{Zizhen Zhang $^{1 *}$ and Yougang Wang ${ }^{1}$}

\section{"Correspondence:}

zzzhaida@163.com

'School of Management Science and Engineering, Anhui University of Finance and Economics, Bengbu, China

\begin{abstract}
In this paper, local stability and Hopf bifurcation of a delayed heroin model with saturated treatment function are discussed. First of all, sufficient conditions for local stability and existence of Hopf bifurcation are obtained by regarding the time delay as a bifurcation parameter and analyzing the distribution of the roots of the associated characteristic equation. Directly afterward, properties of the Hopf bifurcation, such as the direction and stability, are investigated with the aid of the normal form theory and the manifold center theorem. Finally, numerical simulations are presented to justify the obtained theoretical results, and some suggestions are offered for controlling heroin abuse in populations.
\end{abstract}

Keywords: Heroin model; Hopf bifurcation; Stability; Time delay

\section{Introduction}

Heroin is an opiate drug that is synthesized from morphine [1, 2]. It not only causes somatic and psychological effects for heroin users, but also brings social panic and economic loss to the entire human society. Specially, it is one of the most important modes of transmitting infectious diseases such as Human Immunodeficiency Virus (HIV) and Hepatitis C Virus (HCV) [3-6]. In recent years, it has been established that heroin spreads in a population like an epidemic disease. Based on this, some mathematical models have been formulated to describe the epidemic dynamics of heroin users. In [7], Mackintosh and Stewart proposed an exponential model based on the infectious disease model of Kermack and Mckendrick to describe how the use of heroin spreads in an epidemic fashion. White and Comiskey [8] formulated a heroin epidemic model for treating heroin users, and it involves susceptibles, heroin users, and heroin users undergoing treatment, three classes of people. Later, Mulone and Straughan [9] showed that the steady states of the model proposed by White and Comiskey [8] are stable. However, they assumed that the total population is a constant, which is not reasonable in reality. Thus, Wang et al. [10] proposed a heroin model with the bilinear law incidence instead of the standard incidence and studied its stability. Considering the effects of distributed time delay on the dynamics of the heroin model, Samanta et al. [11-14] investigated different forms of heroin models with distributed delays. Motivated by the work of Samanta et al. [11], Abdurahman et al. [15] derived a discretized heroin epidemic model with a distributed time delay and studied its stability and permanence. As stated in [16], all the heroin models above assume that all individuals have the same level of susceptibility. This is not consistent with the reality,

(c) The Author(s) 2019. This article is distributed under the terms of the Creative Commons Attribution 4.0 International License (http://creativecommons.org/licenses/by/4.0/), which permits unrestricted use, distribution, and reproduction in any medium, provided you give appropriate credit to the original author(s) and the source, provide a link to the Creative Commons license, and indicate if changes were made. 
because the individuals at different stages have different levels of susceptibility. Thus, Fang et al. $[16,17]$ investigated the heroin model with age-dependent susceptibility. In addition, there are also some heroin models with nonlinear incidence rate that have received much attention from scholars at home and abroad [18-20].

Obviously, most of the heroin models above assume that the treatment rate of heroin users not in treatment is proportional to the number of them. However, it is only possible to treat a limited number of heroin users at a given time, especially when the medical resources are scarce. Thus, it is more realistic to investigate the heroin model with nonlinear treatment function. To this end, Wangari and Stone [21] formulated the following heroin model with a saturated treatment function:

$$
\left\{\begin{aligned}
\frac{d S(t)}{d t}= & A-\beta U_{1}(t) S(t)-\mu S(t), \\
\frac{d U_{1}(t)}{d t}= & \beta U_{1}(t) S(t)+p U_{2}(t)-\left(\mu+\delta_{1}+\varepsilon\right) U_{1}(t) \\
& -\frac{\alpha U_{1}(t)}{1+\eta U_{1}(t)} \\
\frac{d U_{2}(t)}{d t}= & \frac{\alpha U_{1}(t)}{1+\eta U_{1}(t)}-\left(p+\sigma+\delta_{2}+\mu\right) U_{2}(t), \\
\frac{d U_{3}(t)}{d t}= & \sigma U_{2}(t)+\varepsilon U_{1}(t)-\mu U_{3}(t),
\end{aligned}\right.
$$

where $S(t)$ denotes the number of susceptibles at time $t ; U_{1}(t), U_{2}(t)$, and $U_{3}(t)$ denote the numbers of heroin users not in treatment, heroin users undergoing treatment, and individuals successfully treated from heroin use at time $t$, respectively. $A$ is the constant input rate of the susceptibles through immigration and birth; $\beta$ is the contact rate of the susceptibles with heroin users; $p$ is the rate at which the heroin users undergoing treatment relapse; $\delta_{1}$ and $\delta_{2}$ are the heroin-induced death rates of heroin users not in treatment and heroin users undergoing treatment, respectively; $\varepsilon$ is the self-cure rate of heroin users not in treatment; $\sigma$ is the successful treatment rate of heroin users undergoing treatment; $\alpha$ is the rate at which heroin users are treated; $\eta$ accounts for the extent of saturation of heroin users; $\mu$ is the natural death rate of all the subpopulations. Wangari and Stone [21] studied the stability and backward bifurcations of system (1).

It should be pointed out that Wangari and Stone [21] assume that the heroin users are cured instantaneously, which is not consistent with reality. As stated in [15], it needs some time to become a heroin user for a susceptible one. In exactly the same way, it should also take a period to cure drug users. Therefore, it is more realistic to incorporate the time delay due to the period used to cure heroin users into system (1). We assume that heroin users begin to be treated at $t-\tau$ and they are cured at $t$. Thus, we can obtain the following delayed heroin model:

$$
\left\{\begin{aligned}
\frac{d S(t)}{d t}= & A-\beta U_{1}(t) S(t)-\mu S(t), \\
\frac{d U_{1}(t)}{d t}= & \beta U_{1}(t) S(t)+p U_{2}(t)-\left(\mu+\delta_{1}\right) U_{1}(t) \\
& -\varepsilon U_{1}(t-\tau)-\frac{\alpha U_{1}(t)}{1+\eta U_{1}(t)}, \\
\frac{d U_{2}(t)}{d t}= & \frac{\alpha U_{1}(t)}{1+\eta U_{1}(t)}-\left(p+\delta_{2}+\mu\right) U_{2}(t)-\sigma U_{2}(t-\tau), \\
\frac{d U_{3}(t)}{d t}= & \sigma U_{2}(t-\tau)+\varepsilon U_{1}(t-\tau)-\mu U_{3}(t),
\end{aligned}\right.
$$

where $\tau$ is the period used to cure heroin users. We mainly focus on the effect of the time delay on system (2). 
The organization of this paper is as follows. In Sect. 2, we study local stability and existence of Hopf bifurcation, and sufficient conditions for local stability and existence of Hopf bifurcation are established. Section 3 deals with direction of the Hopf bifurcation, stability, and period of the bifurcating periodic solutions. Computer simulations of the model are performed in Sect. 4. Section 5, which is the last one, contains the conclusions.

\section{Local stability and existence of Hopf bifurcation}

Based on the analysis in [21], we can conclude that if condition $\left(H_{1}\right): R_{0}>1$ and $L_{1}<0$ holds, then system (2) has a unique endemic equilibrium $E_{*}\left(S_{*}, U_{1 *}, U_{2 *}, U_{3 *}\right)$, where

$$
\begin{aligned}
& S_{*}=\frac{A}{\beta U_{1 *}+\mu}, \\
& U_{2 *}=\frac{\alpha U_{1 *}}{\left(p+\sigma+\delta_{2}+\mu\right)\left(1+\eta U_{1 *}\right)}, \\
& U_{3 *}=\frac{\alpha \sigma U_{1 *}+\varepsilon\left(p+\sigma+\delta_{2}+\mu\right)\left(1+\eta U_{1 *}\right) U_{1 *}}{\mu\left(p+\sigma+\delta_{2}+\mu\right)\left(1+\eta U_{1 *}\right)},
\end{aligned}
$$

and $U_{1 *}$ is the positive root of Eq. (3)

$$
L_{2} U_{1 *}^{2}+L_{1} U_{1 *}+L_{0}=0
$$

where

$$
\begin{aligned}
& L_{2}=\left(\mu+\delta_{1}+\varepsilon\right)\left(p+\sigma+\delta_{2}+\mu\right) \beta \eta, \\
& L_{1}=\left(p+\sigma+\delta_{2}+\mu\right)\left[\left(\mu+\delta_{1}+\varepsilon\right)(\beta+\mu \eta)-A \beta \eta\right]+\alpha \beta\left(\sigma+\delta_{2}+\mu\right), \\
& L_{0}=\left[\mu\left(\mu+\delta_{1}+\varepsilon\right)\left(p+\sigma+\delta_{2}+\mu\right)+\alpha \mu\left(\sigma+\delta_{2}+\mu\right)\right]\left(1-R_{0}\right),
\end{aligned}
$$

with

$$
R_{0}=\frac{\beta A\left(p+\sigma+\delta_{2}+\mu\right)}{\mu\left(\mu+\delta_{1}+\varepsilon\right)\left(p+\sigma+\delta_{2}+\mu\right)+\mu \alpha\left(\alpha+\delta_{2}+\sigma\right)} .
$$

The characteristic equation of the linear section of system $(2)$ at $E_{*}\left(S_{*}, U_{1 *}, U_{2 *}, U_{3 *}\right)$ is

$$
\begin{aligned}
\lambda^{4} & +M_{3} \lambda^{3}+M_{2} \lambda^{2}+M_{1} \lambda+M_{0} \\
& +\left(N_{3} \lambda^{3}+N_{2} \lambda^{2}+N_{1} \lambda+N_{0}\right) e^{-\lambda \tau} \\
& +\left(P_{2} \lambda^{2}+P_{1} \lambda+P_{0}\right) e^{-2 \lambda \tau}=0,
\end{aligned}
$$

where

$$
\begin{aligned}
M_{0}= & m_{33} m_{44}\left(m_{11} m_{22}-m_{12} m_{21}\right)-m_{11} m_{23} m_{32} m_{44}, \\
M_{1}= & \left(m_{12} m_{21}-m_{11} m_{22}\right)\left(m_{33}+m_{44}\right)+m_{23} m_{32}\left(m_{11}+m_{44}\right) \\
& -m_{33} m_{44}\left(m_{11}+m_{22}\right), \\
M_{2}= & m_{11} m_{22}+m_{33} m_{44}-m_{12} m_{21}-m_{23} m_{32}
\end{aligned}
$$




$$
\begin{aligned}
& +\left(m_{11}+m_{22}\right)\left(m_{33}+m_{44}\right), \\
M_{3}= & -\left(m_{11}+m_{22}+m_{33}+m_{44}\right), \\
N_{0}= & m_{11} m_{44}\left(m_{33} n_{22}+m_{22} n_{33}\right)-m_{12} m_{21} m_{44} n_{33}, \\
N_{1}= & m_{12} m_{21} n_{33}-n_{22}\left(m_{11} m_{33}+m_{11} m_{44}+m_{33} m_{44}\right) \\
& -n_{33}\left(m_{11} m_{22}+m_{11} m_{44}+m_{22} m_{44}\right), \\
N_{2}= & n_{22}\left(m_{11}+m_{33}+m_{44}\right)+n_{33}\left(m_{11}+m_{22}+m_{44}\right), \\
N_{3}= & -\left(n_{22}+n_{33}\right), \quad P_{0}=m_{11} m_{44} n_{22} n_{33}, \\
P_{1}= & -n_{22} n_{33}\left(m_{11}+m_{44}\right), \quad P_{2}=n_{22} n_{33},
\end{aligned}
$$

and

$$
\begin{aligned}
& m_{11}=-\left(\beta U_{1 *}+\mu\right), \quad m_{12}=-\beta S_{*}, \\
& m_{21}=\beta U_{1 *}, \quad m_{22}=\beta S_{*}-\left(\mu+\delta_{1}\right)-\frac{\alpha}{1+\eta U_{1 *}}, \quad m_{23}=p, \\
& m_{32}=\frac{\alpha}{1+\eta U_{1 *}}, \quad m_{33}=-\left(p+\delta_{2}+\mu\right), \\
& m_{42}=-\mu, \quad n_{22}=-\varepsilon, \quad n_{33}=-\sigma, \quad n_{42}=\varepsilon, \quad n_{43}=\sigma .
\end{aligned}
$$

Multiplying by $e^{\lambda \tau}$, Eq. (4) becomes

$$
\begin{aligned}
& N_{3} \lambda^{3}+N_{2} \lambda^{2}+N_{1} \lambda+N_{0} \\
& +\left(\lambda^{4}+M_{3} \lambda^{3}+M_{2} \lambda^{2}+M_{1} \lambda+M_{0}\right) e^{\lambda \tau} \\
& +\left(P_{2} \lambda^{2}+P_{1} \lambda+P_{0}\right) e^{-\lambda \tau}=0 .
\end{aligned}
$$

When $\tau=0$, Eq. (5) becomes

$$
\lambda^{4}+M_{03} \lambda^{3}+M_{02} \lambda^{2}+M_{01} \lambda+M_{00}=0,
$$

where

$$
\begin{array}{ll}
M_{00}=M_{0}+N_{0}+P_{0}, & M_{01}=M_{1}+N_{1}+P_{1}, \\
M_{02}=M_{2}+N_{2}+P_{2}, & M_{03}=M_{3}+N_{3} .
\end{array}
$$

Based on the Routh-Hurwitz theorem, it can be concluded that all the roots of Eq. (6) have negative real parts if $\left(H_{2}\right)$ holds

$$
\left(H_{2}\right): M_{00}>0, \quad M_{01}>0, \quad M_{03}>0, \quad M_{01} M_{02} M_{03}>M_{01}^{2}+M_{03}^{2} M_{00} .
$$

For $\tau>0$, let $\lambda=i \omega(\omega>0)$ be a root of Eq. (5). Then

$$
\left\{\begin{array}{l}
P_{1}(\omega) \cos \tau \omega-P_{2}(\omega) \sin \tau \omega=P_{3}(\omega) \\
P_{4}(\omega) \sin \tau \omega+P_{5}(\omega) \cos \tau \omega=P_{6}(\omega)
\end{array}\right.
$$


where

$$
\begin{aligned}
& P_{1}(\omega)=\omega^{4}-\left(M_{2}+P_{2}\right) \omega^{2}+M_{0}+P_{0}, \\
& P_{2}(\omega)=\left(M_{1}-P_{1}\right) \omega-M_{3} \omega^{3}, \\
& P_{3}(\omega)=N_{2} \omega^{2}-N_{0}, \\
& P_{4}(\omega)=\omega^{4}-\left(M_{2}-P_{2}\right) \omega^{2}+M_{0}-P_{0}, \\
& P_{5}(\omega)=\left(M_{1}+P_{1}\right) \omega-M_{3} \omega^{3}, \\
& P_{6}(\omega)=N_{3} \omega^{3}-N_{1} \omega .
\end{aligned}
$$

Thus, one can obtain

$$
\begin{aligned}
& \cos \tau \omega=\frac{P_{01}(\omega)}{P_{00}(\omega)}, \\
& \sin \tau \omega=\frac{P_{02}(\omega)}{P_{00}(\omega)},
\end{aligned}
$$

with

$$
\begin{aligned}
P_{00}(\omega)= & \omega^{8}-2 M_{2} \omega^{6}+\left(M_{2}^{2}+2 M_{0}-P_{2}^{2}\right) \omega^{4} \\
& +2\left(P_{0} P_{2}-M_{0} M_{2}\right) \omega \omega^{2}+M_{0}^{2}-P_{0}^{2}, \\
P_{01}(\omega)= & \left(N_{2}-M_{3} N_{3}\right) \omega^{6} \\
& +\left[N_{3}\left(M_{1}-P_{1}\right)-N_{2}\left(M_{2}-P_{2}\right)+M_{3} N_{1}-N_{0}\right] \omega^{4} \\
& +\left[N_{0}\left(M_{2}-P_{2}\right)-N_{1}\left(M_{1}-P_{1}\right)+N_{2}\left(M_{0}-P_{0}\right)\right] \omega^{2} \\
& -N_{0}\left(M_{0}-P_{0}\right), \\
P_{02}(\omega)= & N_{3} \omega^{7}-\left[N_{3}\left(M_{2}+P_{2}\right)+N_{1}\right] \omega^{5} \\
& +\left[N_{3}\left(M_{0}+P_{0}\right)-N_{1}\left(M_{2}+P_{2}\right)\right] \omega^{3}-N_{1}\left(M_{0}+P_{0}\right) \omega .
\end{aligned}
$$

Then we can obtain the following equation with respect to $\omega$ :

$$
P_{01}^{2}(\omega)+P_{02}^{2}(\omega)-P_{00}^{2}(\omega)=0 .
$$

Next, we suppose that $\left(H_{3}\right)$ : Eq. (7) has at least one positive root $\omega_{0}$.

Then Eq. (5) has a pair of purely imaginary roots $\pm i \omega_{0}$. For $\omega_{0}$, we can obtain the corresponding critical value of time delay as follows:

$$
\tau_{0}=\frac{1}{\omega_{0}} \arccos \left\{\frac{P_{01}\left(\omega_{0}\right)}{P_{00}\left(\omega_{0}\right)}\right\} .
$$

In what follows, we differentiate both sides of Eq. (7) with respect to $\tau$ and obtain

$$
\left[\frac{d \lambda}{d \tau}\right]^{-1}=-\frac{G_{1}(\lambda)}{G_{2}(\lambda)}-\frac{\tau}{\lambda}
$$


where

$$
\begin{aligned}
G_{1}(\lambda)= & 3 B_{3} \lambda^{2}+2 B_{2} \lambda+B_{1} \\
& +\left(4 \lambda^{3}+3 M_{3} \lambda^{2}+2 M_{2} \lambda+M_{1}\right) e^{\lambda \tau}+\left(2 P_{2} \lambda+P_{1}\right) e^{-\lambda \tau}, \\
G_{2}(\lambda)= & \lambda\left(\lambda^{4}+M_{3} \lambda^{3}+M_{2} \lambda^{2}+M_{1} \lambda+M_{0}\right) e^{\lambda \tau} \\
& -\lambda\left(P_{2} \lambda^{2}+P_{1} \lambda+P_{0}\right) e^{-\lambda \tau} .
\end{aligned}
$$

Then we can obtain the expression of $\operatorname{Re}\left[\frac{d \lambda}{d \tau}\right]_{\tau=\tau_{0}}^{-1}$. Let

$$
\operatorname{Re}\left[\frac{d \lambda}{d \tau}\right]_{\tau=\tau_{0}}^{-1}=\frac{P_{R} Q_{R}+P_{I} Q_{I}}{Q_{R}^{2}+Q_{I}^{2}}
$$

where

$$
\begin{aligned}
& P_{R}=N_{1}-3 N_{3} \omega^{2}+\left(M_{1}+P_{1}-3 M_{3} \omega^{2}\right) \cos \tau_{0} \omega_{0}-2\left(M_{2} \omega-P_{2} \omega+2 \omega^{3}\right) \sin \tau_{0} \omega_{0}, \\
& P_{I}=-2 N_{2} \omega-2\left(M_{2} \omega+P_{2} \omega-2 \omega^{3}\right) \cos \tau_{0} \omega_{0}-\left(M_{1}-P_{1}-3 M_{3} \omega^{2}\right) \sin \tau_{0} \omega_{0}, \\
& Q_{R}=\left(M_{1} \omega-P_{1} \omega-M_{3} \omega^{3}\right) \cos \tau_{0} \omega_{0}+\left(M_{0}+P_{0}-M_{2} \omega^{2}-P_{2} \omega^{2}+\omega^{4}\right) \sin \tau_{0} \omega_{0}, \\
& Q_{I}=\left(M_{0}-P_{0}-M_{2} \omega^{2}+P_{2} \omega^{2}+\omega^{4}\right) \cos \tau_{0} \omega_{0}-\left(M_{1} \omega+P_{1} \omega-M_{3} \omega^{3}\right) \sin \tau_{0} \omega_{0} .
\end{aligned}
$$

Obviously, if $\left(H_{4}\right): P_{R} Q_{R}+P_{I} Q_{I} \neq 0$ holds, then $\operatorname{Re}\left[\frac{d \lambda}{d \tau}\right]_{\tau=\tau_{0}}^{-1} \neq 0$. Based on the discussion above and the Hopf bifurcation theorem in [22], we have the following results.

Theorem 1 For system (2), if conditions $\left(H_{1}\right)-\left(H_{4}\right)$ hold, then the positive equilibrium $E_{*}\left(S_{*}, U_{1 *}, U_{2 *}, U_{3 *}\right)$ is locally asymptotically stable when $\tau \in\left[0, \tau_{0}\right)$; system (2) undergoes a Hopf bifurcation at $E_{*}\left(S_{*}, U_{1 *}, U_{2 *}, U_{3 *}\right)$ when $\tau=\tau_{0}$, and a family of periodic solutions bifurcate from $E_{*}\left(S_{*}, U_{1 *}, U_{2 *}, U_{3 *}\right)$.

\section{Direction and stability of Hopf bifurcation}

In this section, the formulae for determining the direction and stability of the Hopf bifurcation of system (2) at $\tau=\tau_{0}$ are presented. Let $\tau=\tau_{0}+\mu_{0}, \mu_{0} \in R$, so that $\mu_{0}=0$ is the Hopf bifurcation value for the system. Define the space of continuous real-valued functions as $C=C\left([-1,0], R^{4}\right)$. Let $u_{1}(t)=S(t)-S_{*}, u_{2}(t)=U_{1}(t)-U_{1 *}, u_{3}(t)=U_{2}(t)-U_{2 *}$, $u_{4}(t)=U_{3}(t)-U_{3 *}$, and normalize time delay with the scaling $t \rightarrow(t / \tau)$. Then the delay system (2) transforms to a functional differential equation in $C$ as follows:

$$
\dot{u}(t)=L_{\mu_{0}}\left(u_{t}\right)+F\left(\mu_{0}, u_{t}\right)
$$

where $u(t)=\left(u_{1}, u_{2}, u_{3}, u_{4}\right)^{T} \in C=C\left([-1,0], R^{4}\right)$ and $L_{\mu}: C \rightarrow R^{4}$ and $F: R \times C \rightarrow R^{4}$ are given as follows:

$$
L_{\mu_{0}} \phi=\left(\tau_{0}+\mu_{0}\right)\left(M_{\max } \phi(0)+N_{\max } \phi(-1)\right)
$$


and

$$
F\left(\mu_{0}, \phi\right)=\left(\tau_{0}+\mu_{0}\right)\left(\begin{array}{c}
0 \\
m_{25} \phi_{2}^{2}(0)+m_{26} \phi_{2}^{3}(0)+\cdots \\
m_{35} \phi_{2}^{2}(0)+m_{36} \phi_{2}^{3}(0)+\cdots \\
0
\end{array}\right)
$$

with

$$
\begin{aligned}
M_{\max } & =\left(\begin{array}{cccc}
m_{11} & m_{12} & 0 & 0 \\
m_{21} & m_{22} & m_{23} & 0 \\
0 & m_{32} & m_{33} & 0 \\
0 & 0 & 0 & m_{44}
\end{array}\right), \\
N_{\max } & =\left(\begin{array}{cccc}
0 & 0 & 0 & 0 \\
0 & n_{22} & 0 & 0 \\
0 & 0 & n_{33} & 0 \\
0 & n_{42} & n_{43} & 0
\end{array}\right)
\end{aligned}
$$

and

$$
\begin{array}{ll}
m_{25}=\frac{\alpha \eta}{\left(1+\eta U_{1 *}\right)^{3}}, & m_{26}=-\frac{\alpha \eta^{2}}{\left(1+\eta U_{1 *}\right)^{4}}, \\
m_{35}=-\frac{\alpha \eta}{\left(1+\eta U_{1 *}\right)^{3}}, & m_{36}=\frac{\alpha \eta^{2}}{\left(1+\eta U_{1 *}\right)^{4}} .
\end{array}
$$

By the Riesz representation theorem, there exists a function $\eta\left(\theta, \mu_{0}\right)$ for $\theta \in[-1,0]$ such that

$$
L_{\mu_{0}} \phi=\int_{-1}^{0} d \eta\left(\theta, \mu_{0}\right) \phi(\theta)
$$

for $\phi \in C$. In fact, we choose

$$
\eta\left(\theta, \mu_{0}\right)=\left(\tau_{0}+\mu_{0}\right)\left(M_{\max } \delta(\theta)+N_{\max } \delta(\theta+1)\right)
$$

where $\delta(\theta)$ is the Dirac delta function.

$$
\text { For } \phi \in C\left([-1,0], R^{4}\right) \text {, define }
$$

$$
A\left(\mu_{0}\right) \phi= \begin{cases}\frac{d \phi(\theta)}{d \theta}, & -1 \leq \theta<0 \\ \int_{-1}^{0} d \eta\left(\theta, \mu_{0}\right) \phi(\theta), & \theta=0\end{cases}
$$

and

$$
R\left(\mu_{0}\right) \phi= \begin{cases}0, & -1 \leq \theta<0, \\ F\left(\mu_{0}, \phi\right), & \theta=0 .\end{cases}
$$

Then system (9) is equivalent to

$$
\dot{u}(t)=A\left(\mu_{0}\right) u_{t}+R\left(\mu_{0}\right) u_{t} .
$$


For $\varphi \in C^{1}\left([0,1],\left(R^{4}\right)^{*}\right)$, the adjoint operator $A^{*}$ of $A(0)$ is defined as follows:

$$
A^{*}(\varphi)= \begin{cases}-\frac{d \varphi(s)}{d s}, & 0<s \leq 1 \\ \int_{-1}^{0} d \eta^{T}(s, 0) \varphi(-s), & s=0\end{cases}
$$

Next, we define the bilinear inner form for $A$ and $A^{*}$ :

$$
\langle\varphi(s), \phi(\theta)\rangle=\bar{\varphi}(0) \phi(0)-\int_{\theta=-1}^{0} \int_{\xi=0}^{\theta} \bar{\varphi}(\xi-\theta) d \eta(\theta) \phi(\xi) d \xi
$$

where $\eta(\theta)=\eta(\theta, 0)$.

Suppose that $q(\theta)=\left(1, q_{2}, q_{3}, q_{4}\right)^{T} e^{i \omega_{0} \tau_{0} \theta}$ is the eigenvector of $A(0)$ corresponding to $i \omega_{0} \tau_{0}$ and $q^{*}(s)=D\left(1, q_{2}^{*}, q_{3}^{*}, q_{4}^{*}\right)^{T} e^{i \tau_{0} \omega_{0} s}$ is the eigenvector of $A^{*}(0)$ corresponding to $-i \tau_{0} \omega_{0}$. Then we can obtain

$$
\begin{aligned}
& q_{2}=\frac{i \omega_{0}-m_{11}}{m_{12}}, \\
& q_{3}=\frac{i \omega_{0}-m_{22}-n_{22} e^{-i \tau_{0} \omega_{0}}}{m_{23}}, \\
& q_{4}=\frac{n_{42} q_{2}+n_{43} q_{3}}{i \omega_{0}-m_{44}}, \\
& q_{2}^{*}=-\frac{i \omega_{0}+m_{11}}{m_{21}}, \\
& q_{3}^{*}=\frac{\Delta_{2}-m_{42} e^{i \tau_{0} \omega_{0}}}{m_{32}}, \\
& q_{4}^{*}=-\frac{m_{23} m_{32} q_{2}}{\Delta_{1}\left(\Delta_{2}-n_{42} e^{i \tau_{0} \omega_{0}}\right)+m_{32} n_{43} e^{i \tau_{0} \omega_{0}}}, \\
& \Delta_{1}=i \omega_{0}+m_{33}+n_{33} e^{i \tau_{0} \omega_{0}}, \\
& \Delta_{2}=-\left(i \omega_{0}+m_{22}+n_{22} e^{i \tau_{0} \omega_{0}}\right) q_{2}-m_{12} .
\end{aligned}
$$

From Eq. (13), we can obtain

$$
\begin{aligned}
\bar{D}= & {\left[1+q_{2} q_{2}^{*}+q_{3} \bar{q}_{3}^{*}+q_{4} \bar{q}_{4}^{*}\right.} \\
& \left.+\tau_{0} e^{-i \tau_{0} \omega_{0}}\left(q_{2}\left(n_{22} \bar{q}_{2}^{*}+n_{42} \bar{q}_{4}^{*}\right)+q_{3}\left(n_{33} \bar{q}_{3}^{*}+n_{43} \bar{q}_{4}^{*}\right)\right)\right]^{-1}
\end{aligned}
$$

such that $\left\langle q^{*}, q\right\rangle=1$ and $\left\langle q^{*}, \bar{q}\right\rangle=0$.

Next, based on the algorithms in [22] and a computation process similar to that in [2325], we can obtain

$$
\begin{aligned}
g_{20}= & 2 \bar{D} \tau_{0}\left(m_{25} \bar{q}_{2}^{*} q_{2}^{2}+m_{35} \bar{q}_{3}^{*} q_{2}^{2}\right), \\
g_{11}= & 2 \bar{D} \tau_{0} q_{2} \bar{q}_{2}\left(m_{25} \bar{q}_{2}^{*}+m_{35} \bar{q}_{3}^{*}\right), \\
g_{02}= & 2 \bar{D} \tau_{0}\left(m_{25} \bar{q}_{2}^{*} \bar{q}_{2}^{2}+m_{35} \bar{q}_{3}^{*} \bar{q}_{2}^{2}\right), \\
g_{21}= & 2 \bar{D} \tau_{0}\left[\bar{q}_{2}^{*}\left(m_{25}\left(2 W_{11}^{(2)}(0) q_{2}+W_{20}^{(2)}(0) \bar{q}_{2}\right)+3 m_{26} q_{2}^{2} \bar{q}_{2}\right)\right. \\
& \left.+\bar{q}_{3}^{*}\left(m_{35}\left(2 W_{11}^{(2)}(0) q_{2}+W_{20}^{(2)}(0) \bar{q}_{2}\right)+3 m_{36} q_{2}^{2} \bar{q}_{2}\right)\right],
\end{aligned}
$$


with

$$
\begin{aligned}
& W_{20}(\theta)=\frac{i g_{20} q(0)}{\tau_{0} \omega_{0}} e^{i \tau_{0} \omega_{0} \theta}+\frac{i \bar{g}_{02} \bar{q}(0)}{3 \tau_{0} \omega_{0}} e^{-i \tau_{0} \omega_{0} \theta}+E_{1} e^{2 i \tau_{0} \omega_{0} \theta}, \\
& W_{11}(\theta)=-\frac{i g_{11} q(0)}{\tau_{0} \omega_{0}} e^{i \tau_{0} \omega_{0} \theta}+\frac{i \bar{g}_{11} \bar{q}(0)}{\tau_{0} \omega_{0}} e^{-i \tau_{0} \omega_{0} \theta}+E_{2} .
\end{aligned}
$$

$E_{1}$ and $E_{2}$ can be obtained by the following two equations:

$$
\begin{aligned}
E_{1} & =2\left(\begin{array}{cccc}
2 i \omega_{0}-m_{11} & -m_{12} & 0 & 0 \\
-m_{21} & m_{22}^{\prime} & -m_{23} & 0 \\
0 & -m_{32} & m_{33}^{\prime} & 0 \\
0 & -n_{42} e^{-2 i \tau_{0} \omega_{0}} & -n_{43} e^{-2 i \tau_{0} \omega_{0}} & 2 i \omega_{0}-m_{44}
\end{array}\right)^{-1} \times\left(\begin{array}{c}
0 \\
m_{25} q_{2}^{2} \\
m_{35} q_{2}^{2} \\
0
\end{array}\right), \\
E_{2} & =-\left(\begin{array}{cccc}
m_{11} & m_{12} & 0 & 0 \\
m_{21} & m_{22}+n_{22} & m_{23} & 0 \\
0 & m_{32} & m_{33}+n_{33} & 0 \\
0 & n_{42} & n_{43} & m_{44}
\end{array}\right)^{-1} \times\left(\begin{array}{c}
0 \\
2 m_{25} q_{2} \bar{q}_{2} \\
2 m_{35} q_{2} \bar{q}_{2} \\
0
\end{array}\right),
\end{aligned}
$$

with

$$
\begin{aligned}
& m_{22}^{\prime}=2 i \omega_{0}-m_{22}-n_{22} e^{-2 i \tau_{0} \omega_{0}}, \\
& m_{33}^{\prime}=2 i \omega_{0}-m_{33}-n_{33} e^{-2 i \tau_{0} \omega_{0}} .
\end{aligned}
$$

Then one can obtain

$$
\begin{aligned}
& C_{1}(0)=\frac{i}{2 \tau_{0} \omega_{0}}\left(g_{11} g_{20}-2\left|g_{11}\right|^{2}-\frac{\left|g_{02}\right|^{2}}{3}\right)+\frac{g_{21}}{2} \\
& \mu_{2}=-\frac{\operatorname{Re}\left\{C_{1}(0)\right\}}{\operatorname{Re}\left\{\lambda^{\prime}\left(\tau_{0}\right)\right\}}, \\
& \beta_{2}=2 \operatorname{Re}\left\{C_{1}(0)\right\}, \\
& T_{2}=-\frac{\operatorname{Im}\left\{C_{1}(0)\right\}+\mu_{2} \operatorname{Im}\left\{\lambda^{\prime}\left(\tau_{0}\right)\right\}}{\tau_{0} \omega_{0}} .
\end{aligned}
$$

In conclusion, we have the following results.

Theorem 2 For system (2), if $\mu_{2}>0\left(\mu_{2}<0\right)$, then the Hopf bifurcation is supercritical (subcritical); if $\beta_{2}<0\left(\beta_{2}>0\right)$, then the bifurcating periodic solutions are stable (unstable); if $T_{2}>0\left(T_{2}<0\right)$, then the period of the bifurcating periodic solutions increases (decreases).

\section{Numerical simulation}

In this section, some numerical simulations are performed to verify analytically obtained results. We choose the same set of parameters as those in [21]: $A=3, P=0.46, \alpha=0.9$, $\beta=0.001, \delta_{1}=0.002, \delta_{2}=0.001, \sigma=0,1, \varepsilon=0.015, \eta=0.15$, and $\mu=0.01$. Then we obtain 
the following specific case of system (2):

$$
\left\{\begin{aligned}
& \frac{d S(t)}{d t}=3-0.001 U_{1}(t) S(t)-0.01 S(t), \\
& \frac{d U_{1}(t)}{d t}= 0.001 U_{1}(t) S(t)+0.46 U_{2}(t)-0.012 U_{1}(t) \\
&-0.015 U_{1}(t-\tau)-\frac{0.9 U_{1}(t)}{1+0.15 U_{1}(t)}, \\
& \frac{d U_{2}(t)}{d t}= \frac{0.9 U_{1}(t)}{1+0.15 U_{1}(t)}-0.471 U_{2}(t)-0.1 U_{2}(t-\tau), \\
& \frac{d U_{3}(t)}{d t}= 0.1 U_{2}(t-\tau)+0.015 U_{1}(t-\tau)-0.01 U_{3}(t) .
\end{aligned}\right.
$$

Then we obtain $R_{0}=1.4885>1$ and Eq. (3) becomes of the following form:

$$
2.3126 e-006 U_{1 *}^{2}-1.1851 e-004 U_{1 *}-5.5986 e-004=0,
$$

from which one can obtain that system (15) has a unique positive equilibrium $E_{*}$ (45.7321, $55.5995,9.3976,177.3752)$. Further, we can verify that $E_{*}(45.7321,55.5995,9.3976$, 177.3752) is locally asymptotically stable when $\tau=0$.

For $\tau>0$, by using Matlab software package, we obtain $\omega_{0}=0.0368, \tau_{0}=40.1285, P_{R} Q_{R}+$ $P_{I} Q_{I}=0.0082>0$. In other words, the conditions for the occurrence of the Hopf bifurcation are satisfied for system (15). According to Theorem 1, we can conclude that the unique positive equilibrium $E_{*}(45.7321,55.5995,9.3976,177.3752)$ is locally asymptotically stable when $\tau \in\left[0, \tau_{0}=40.1285\right)$. This can be illustrated by Figs. $1-2$. System (2) undergoes a Hopf bifurcation at $E_{*}(45.7321,55.5995,9.3976,177.3752)$ when $\tau=\tau_{0}=40.1285$ and a family of periodic solutions bifurcate from $E_{*}(45.7321,55.5995,9.3976,177.3752)$, which can be shown as in Figs. 3-4. The bifurcation phenomenon can be also illustrated by the bifurcation diagrams in Fig. 5. Moreover, by some complex computations, we obtain $C_{1}(0)=-0.0462+0.0029 i, \lambda^{\prime}\left(\tau_{0}\right)=0.3729-0.1846 i, \mu_{2}=0.1239>0, \beta_{2}=-0.0924<0$, and $T_{2}=0.0135>0$. Based on Theorem 2, we know that the Hopf bifurcation is supercritical, the periodic solutions are stable and increase.

In addition, according to numerical simulations, we find that: (i) the number of susceptibles decreases and the numbers of the other three populations increase when the number
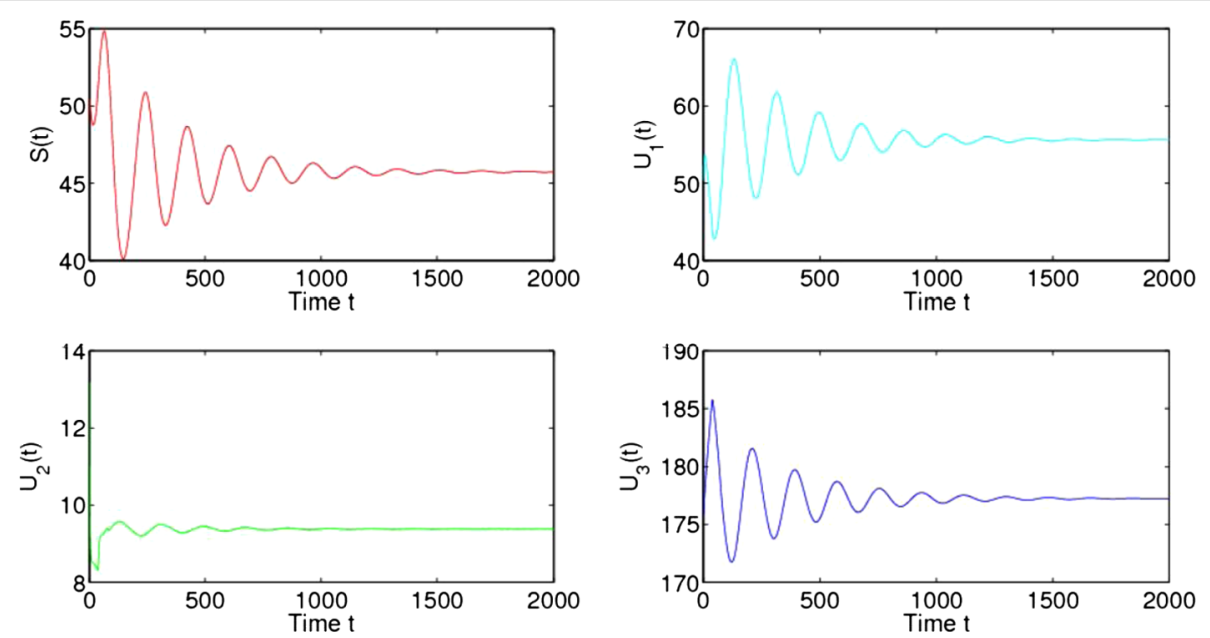

Figure 1 Time plots of $S, U_{1}, U_{2}$ and $U_{3}$ with $\tau=37.45<\tau_{0}=40.1285$ 


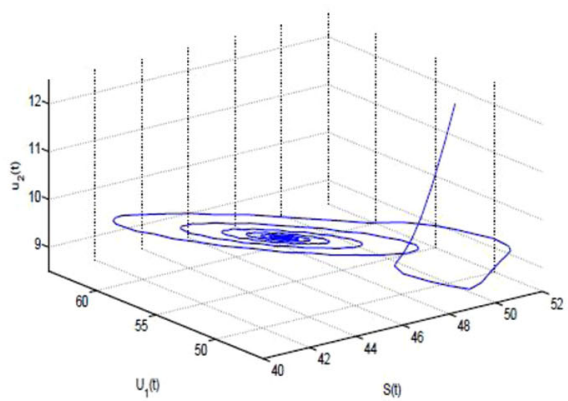

(a)

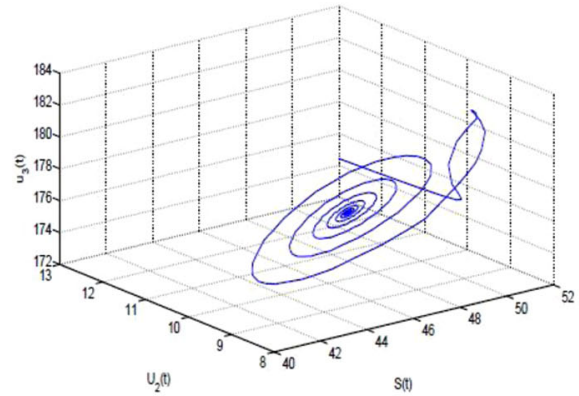

(c)

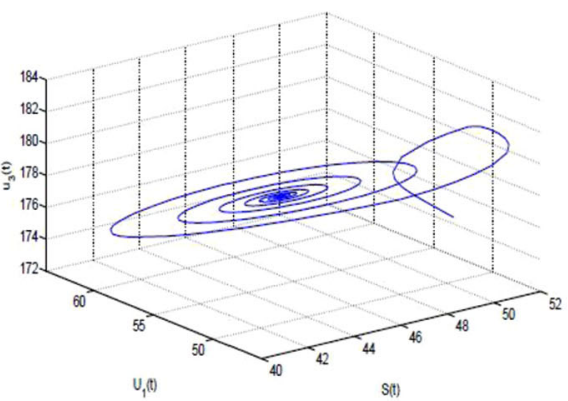

(b)

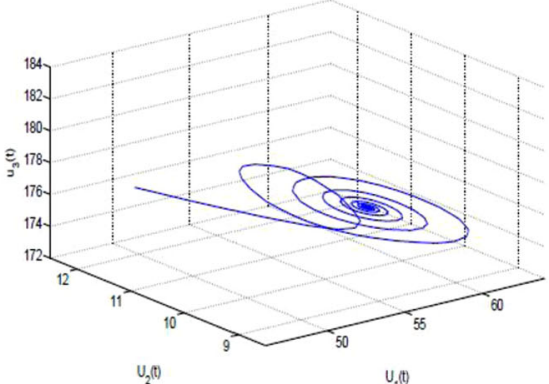

(d)

Figure 2 Phase portrait of system (15) with $\tau=37.45<\tau_{0}=40.1285$ : (a) $S-U_{1}-U_{2}$, (b) $S-U_{1}-U_{3}$, (c) $S-U_{2}-U_{3}$, (d) $U_{1}-U_{2}-U_{3}$
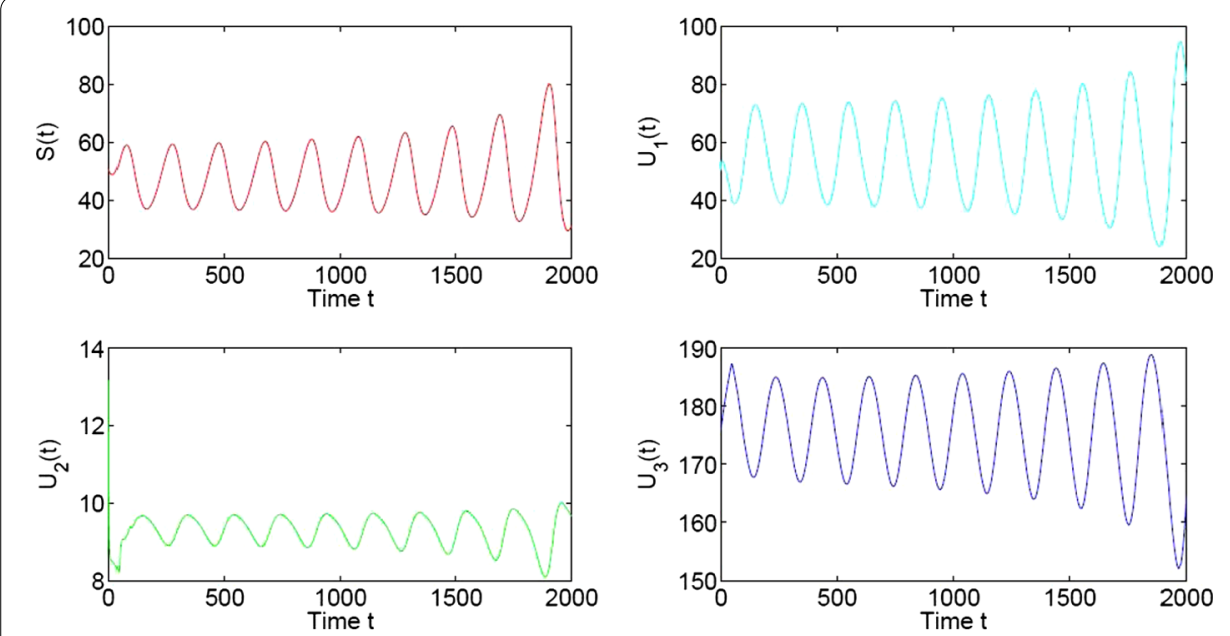

Figure 3 Time plots of $S, U_{1}, U_{2}$ and $U_{3}$ with $\tau=45.25>\tau_{0}=40.1285$

of $A$ or $\beta$ in system (15) increases; this can be demonstrated by Figs. 6-7. (ii) the numbers of susceptibles and heroin users not in treatment decrease; nevertheless, the numbers of heroin users undergoing treatment and individuals successfully treated from heroin use increase when the number of $\alpha$ in system (15) increases, which can be illustrated by Fig. 8. (iii) the number of heroin users not in treatment increases and the numbers of the 


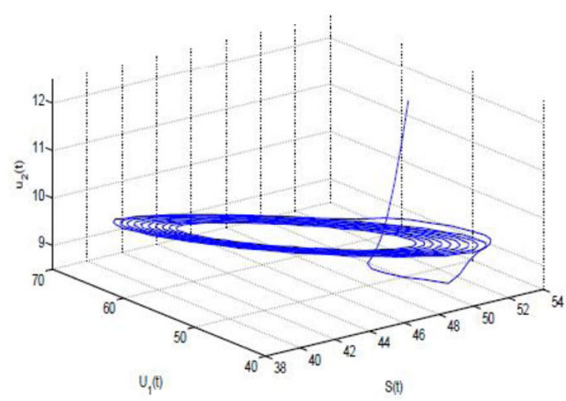

(a)

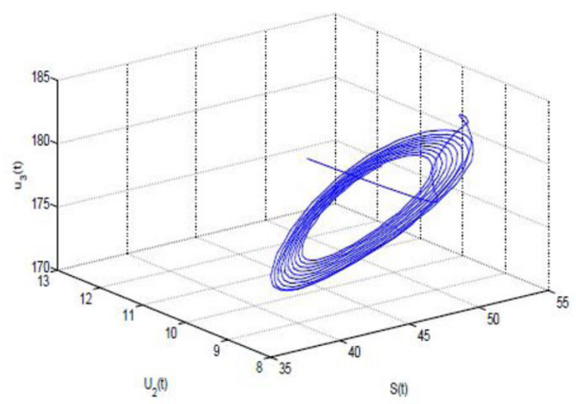

(c)

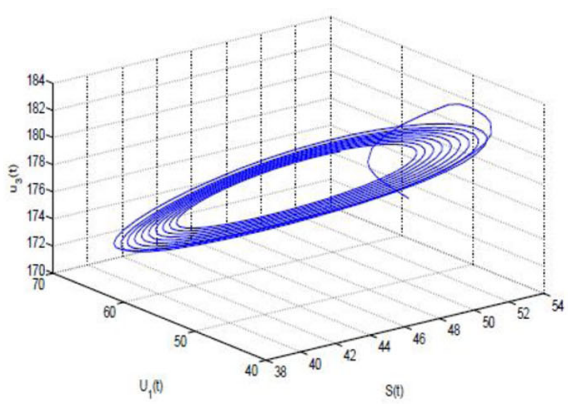

(b)

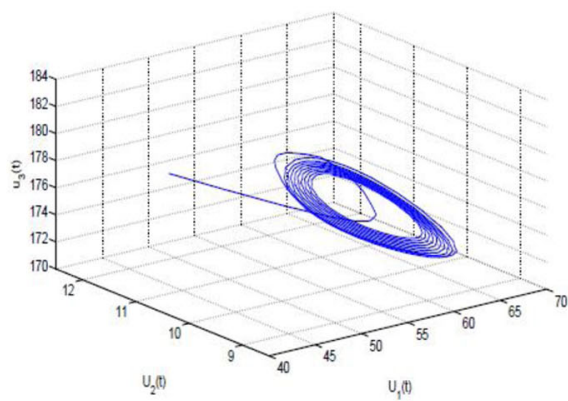

(d)

Figure 4 Phase portrait of system (15) with $\tau=45.25>\tau_{0}=40.1285$ : (a) $S-U_{1}-U_{2}$, (b) $S-U_{1}-U_{3}$, (c) $S-U_{2}-U_{3}$, (d) $U_{1}-U_{2}-U_{3}$

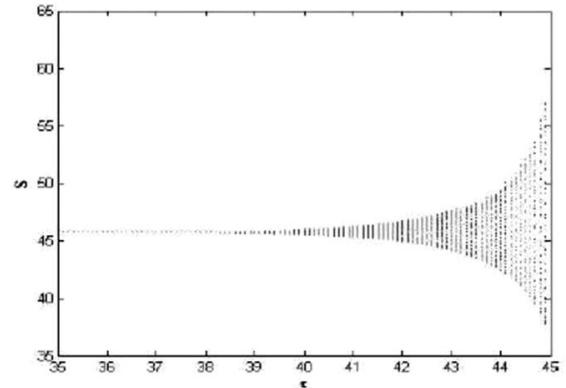

(a)

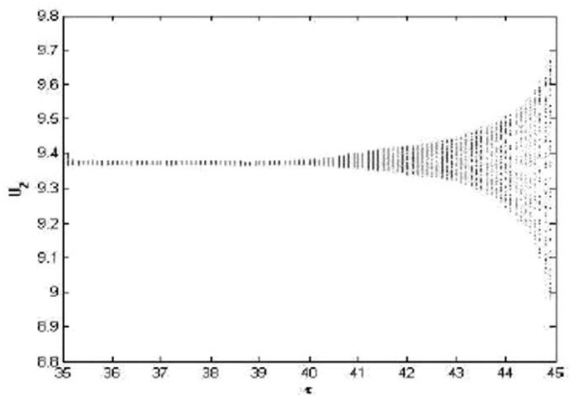

(c)

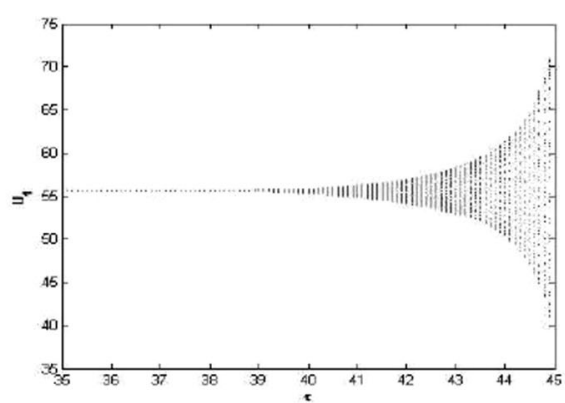

(b)

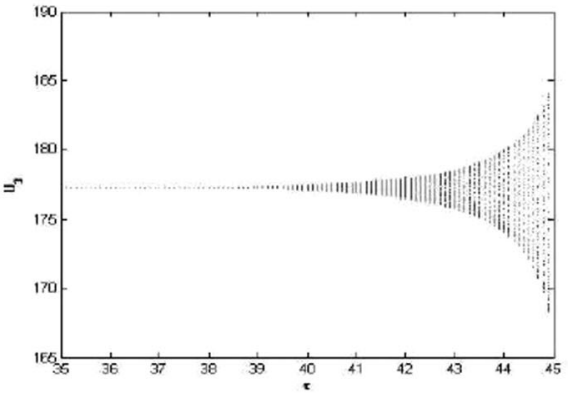

(d)

Figure 5 Bifurcation diagram of system (15) with respect to $\tau$ : (a) $S$, (b) $U_{1}$, (c) $U_{2}$, (d) $U_{3}$ 


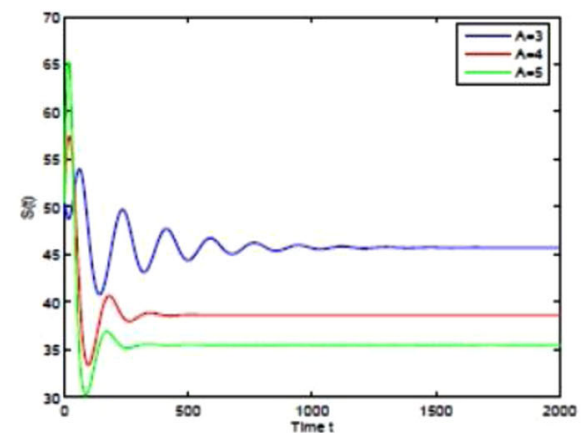

(a)

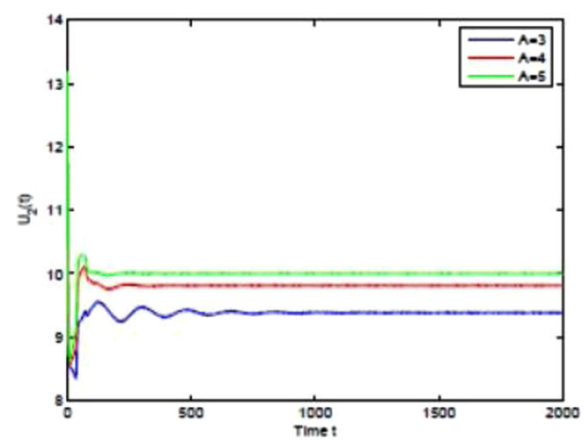

(c)

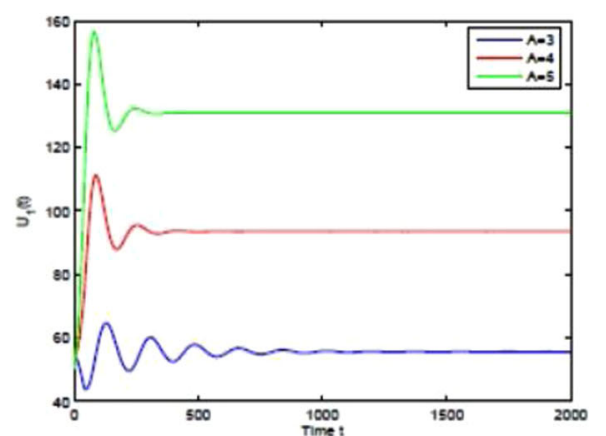

(b)

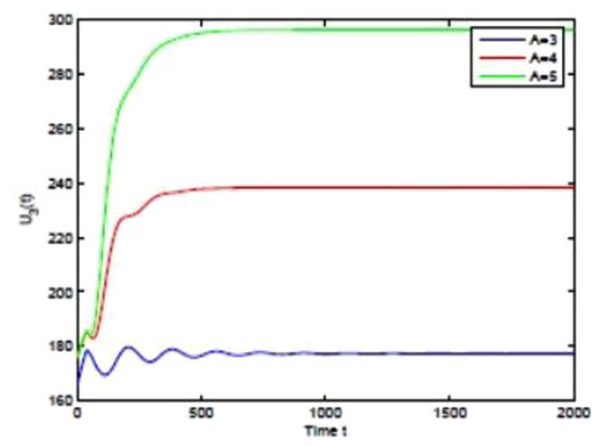

(d)

Figure 6 Effect of $A$ on all classes for $\tau=35.25$ : (a) $S$, (b) $U_{1},(\mathbf{c}) U_{2}$, (d) $U_{3}$

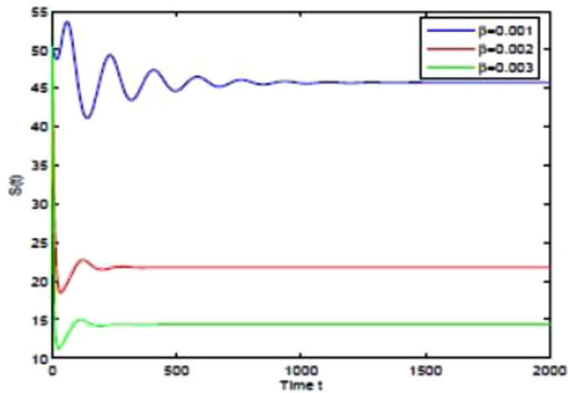

(a)

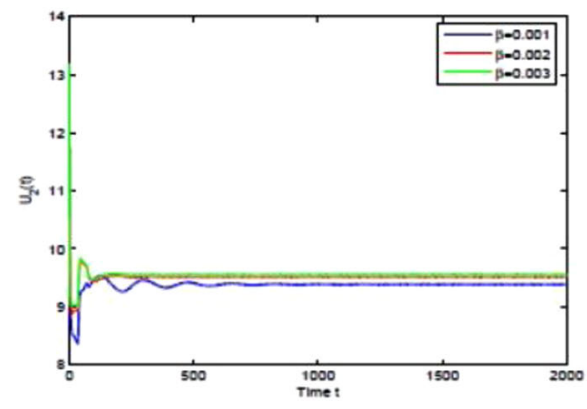

(c)

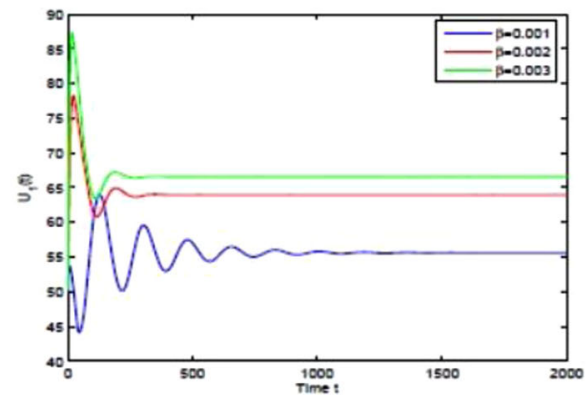

(b)

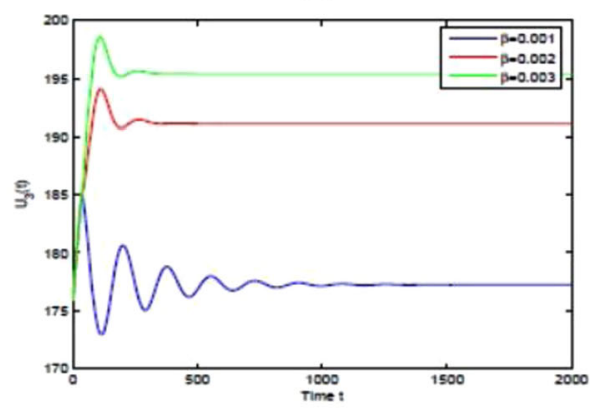

(d)

Figure 7 Effect of $\beta$ on all classes for $\tau=35.25$ : (a) $S$, (b) $U_{1},(\mathbf{c}) U_{2}$, (d) $U_{3}$ 


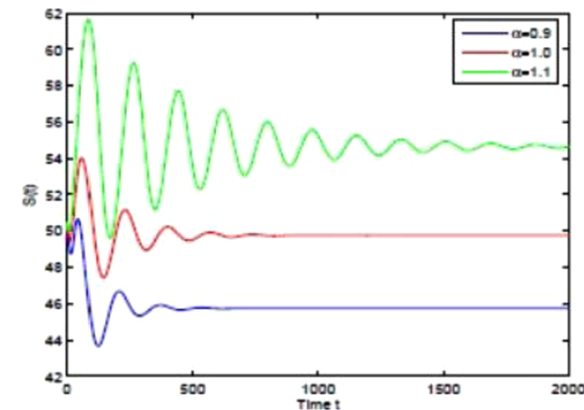

(a)

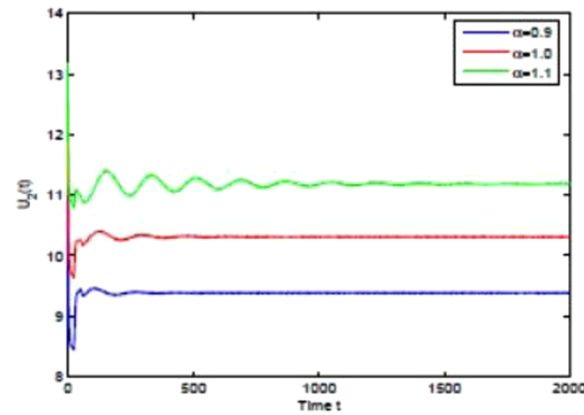

(c)

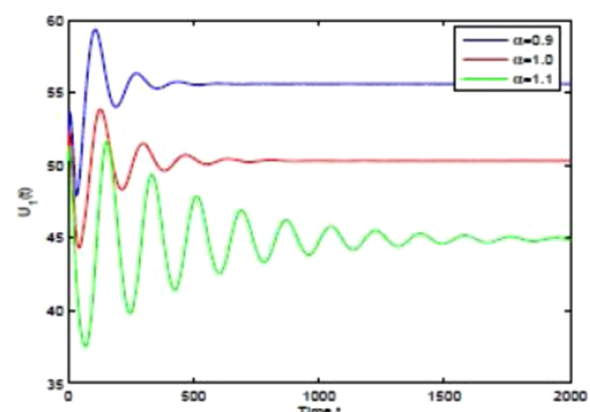

(b)

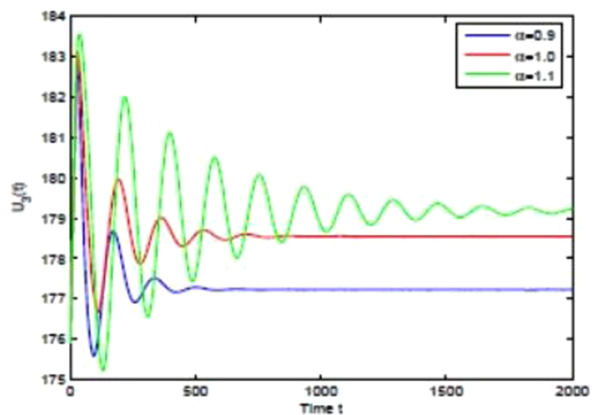

$(d)$

Figure 8 Effect of $\alpha$ on all classes for $\tau=25.25$ : (a) $S,(\mathbf{b}) U_{1},(\mathbf{c}) U_{2},(\mathbf{d}) U_{3}$

other three populations decrease when the number of $\eta$ in system (15) increases. This phenomenon can be shown as in Fig. 9 .

\section{Conclusions}

In this paper, a delayed heroin model with saturated treatment function in the form of $\frac{\alpha U_{1}}{1+\eta U_{1}}$ is discussed, which is different from the model in [21]. In our model, we assume that the heroin users cannot be cured instantaneously and it needs a period to cure heroin users, which is more in line with truth. Compared with the work by Wangari and Stone in [21], we mainly focus on the effect of the time delay on the proposed model in the present paper.

It has been shown that the time delay may destabilize the positive equilibrium of the model and cause the population to fluctuate if certain conditions are satisfied. When the value of the time delay is below the threshold $\tau_{0}$, then the positive equilibrium of the model is locally asymptotically stable. In this case, the heroin abuse among the populations can be controlled. However, when the value of the time delay passes through the threshold $\tau_{0}$, the model will lose its stability and a Hopf bifurcation occurs and a family of periodic solutions bifurcate from the positive equilibrium. In this case, the populations in the model will oscillate in the vicinity of $E_{*}\left(S_{*}, U_{1 *}, U_{2 *}, U_{3 *}\right)$. Namely, the heroin abuse in the populations will be out of control. Furthermore, properties of the Hopf bifurcation are investigated with the aid of the normal form theory and center manifold theorem. Finally, numerical simulations are presented to verify the analytical predictions.

It has been observed in our simulations that the number of heroin users not in treatment increases when the number of $A$ increases. Thus, the input rate of the susceptibles 


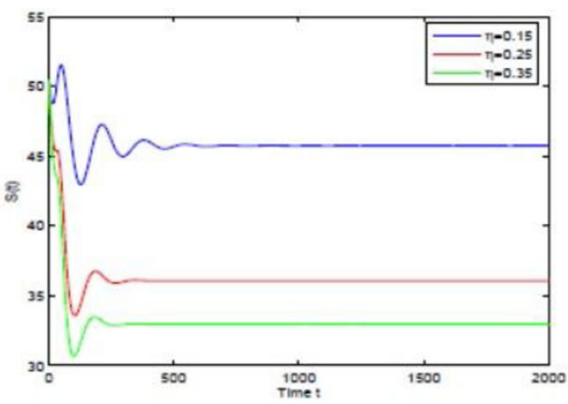

(a)

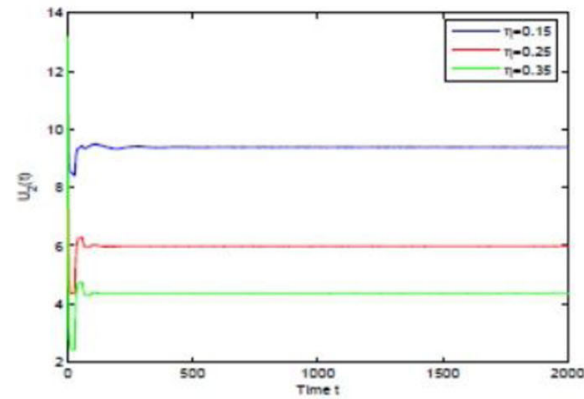

(c)

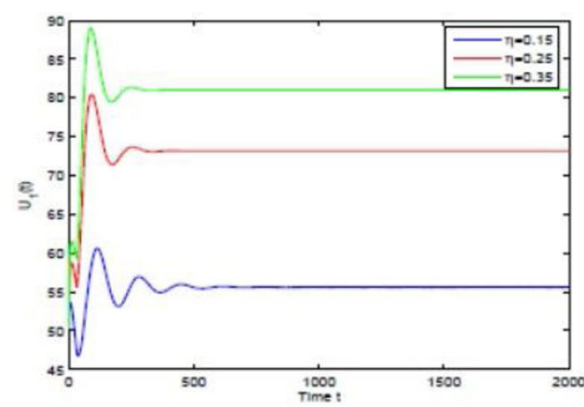

(b)

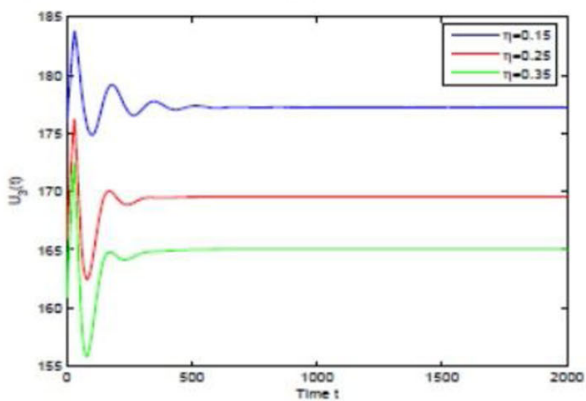

(d)

Figure 9 Effect of $\eta$ on all classes for $\tau=28.25$ : (a) $S$, (b) $U_{1},(\mathbf{c}) U_{2}$, (d) $U_{3}$

in a community should be controlled properly, especially the input rate through immigration. Similarly, it is strongly recommended that people should exercise self-control so as to protect themselves from drugs, since the number of heroin users not in treatment increases when the number of $\beta$ increases. The number of heroin users not in treatment decreases when the number of $\alpha$ increases, which suggests that populations in a community should periodically go to hospital for physical examination and early recognition, treatment. Also, the number of heroin users not in treatment decreases when the number of $\eta$ increases. This gives us a suggestion that we should improve medical facilities continuously and ensure that medical resources are sufficient and available.

\section{Acknowledgements}

The authors are grateful to the editor and the anonymous referees for their valuable comments and suggestions on the paper.

\section{Funding}

This research was supported by the National Natural Science Foundation of China (Nos. 61773181, 11461024), Natural Science Foundation of Anhui Province (Nos. 1608085QF145, 1608085QF151, 1708085MA17), Natural Science Foundation of Inner Mongolia Autonomous Region (No. 2018MS01023), Project of Support Program for Excellent Youth Talent in Colleges and Universities of Anhui Province (Nos. gxyqZD2018044, gxbjZD49), Bengbu University National Research Fund Cultivation Project (2017GJPY03).

Availability of data and materials

All of the authors declare that all the data can be accessed in our manuscript in the numerical simulation section. 


\section{Publisher's Note}

Springer Nature remains neutral with regard to jurisdictional claims in published maps and institutional affiliations.

Received: 10 August 2018 Accepted: 6 February 2019 Published online: 18 February 2019

\section{References}

1. Davis, G.G.: Complete republication: national association of medica examiners position paper: recommendations for the investigation, diagnosis and certification of deaths related to opioid drugs. J. Med. Toxicol. 10, 100-106 (2014)

2. Fang, B., Li, X.Z., Martcheva, M., Cai, L.M.: Global asymptotic properties of a heroin epidemic model with treat-age. Appl. Math. Comput. 263, 315-331 (2015)

3. Liu, J.L., Zhang, T.L.: Global behaviour of a heroin epidemic model with distributed delays. Appl. Math. Lett. 24, 1685-1692 (2011)

4. Garten, R.J., Lai, S., Zhang, J.: Rapid transmission of hepatitis C virus among young injecting heroin users in Southern China. Int. J. Epidemiol. 33, 182-188 (2004)

5. Li, X., Zhou, Y., Stanton, B.: Illicit drug initiation among institutionalized drug users in China. Addict. 97, 575-582 (2002)

6. Cohen, J.: HIV/AIDS in China: poised for take off? Science 304, 1430-1432 (2004)

7. Mackintosh, D., Stewart, G.: A mathematical model of a heroin epidemic: implications for control policies. J. Epidemiol. Community Health 33, 299-304 (1979)

8. White, E., Comiskey, C.: Heroin epidemics, treatment and ODE modelling. Math. Biosci. 208, 312-324 (2007)

9. Mulone, G., Straughan, B.: A note on heroin epidemics. Math. Biosci. 218, 138-141 (2009)

10. Wang, X.Y., Yang, J.Y., Li, X.Z.: Dynamics of a heroin epidemic model with very population. Appl. Math. 2, 732-738 (2011)

11. Samanta, G.P.: Dynamic behaviour for a nonautonomous heroin epidemic model with time delay. J. Appl. Math. Comput. 35, 161-178 (2011)

12. Liu, J.L., Zhang, T.L.: Global behaviour of a heroin epidemic model with distributed delays. Appl. Math. Lett. 24 1685-1692 (2011)

13. Huang, G., Liu, A.P.: A note on global stability for a heroin epidemic model with distributed delay. Appl. Math. Lett. 26 687-691 (2013)

14. Fang, B., Li, X.Z., Martcheva, M., Cai, L.M.: Global stability for a heroin model with two distributed delays. Discrete Contin. Dyn. Syst., Ser. B 19, 715-733 (2017)

15. Abdurahman, X., Zhang, L., Teng, Z.D.: Global dynamics of a discretized heroin epidemic model with time delay. Abstr. Appl. Anal. 2014, Article ID 742385 (2014)

16. Fang, B., Li, X.Z., Martcheva, M., Cai, L.M.: Global stability for a heroin model with age-dependent susceptibility. J. Syst. Sci. Complex. 28, 1243-1257 (2015)

17. Fang, B., Li, X.Z., Martcheva, M., Cai, L.M.: Global asymptotic properties of a heroin epidemic model with treat-age. Appl. Math. Comput. 263, 315-331 (2015)

18. Ma, M.J., Liu, S.Y., Li, J.: Bifurcation of a heroin model with nonlinear incidence rate. Nonlinear Dyn. 88, 555-565 (2017)

19. Djilali, S., Touaoula, T.M., Miri, S.E.H.: A heroin epidemic model: very general non linear incidence, treat-age, and global stability. Acta Appl. Math. 152, 171-194 (2017)

20. Yang, J.Y., Li, X.X., Zhang, F.Q.: Global dynamics of a heroin epidemic mode I with age structure and nonlinear incidence. Int. J. Biomath. 2016, Article ID 1650033 (2016)

21. Wangari, I.M., Stone, L.: Analysis of a heroin epidemic model with saturated treatment function. J. Appl. Math. 2017, Article ID 1953036 (2017)

22. Hassard, B.D., Kazarinoff, N.D., Wan, Y.H.: Theory and Applications of Hopf Bifurcation. Cambridge University Press, Cambridge (1981)

23. Xu, C.J.: Delay-induced oscillations in a competitor-competitor-mutualist Lotka-Volterra model. Complexity 2017 Article ID 2578043 (2017)

24. Zhao, T., Bi, D.J.: Hopf bifurcation of a computer virus spreading model in the network with limited anti-virus ability. Adv. Differ. Equ. 2017, Article ID 183 (2017)

25. Zhang, Z.Z., Bi, D.J.: Bifurcation analysis in a delayed computer virus model with the effect of external computers. Adv. Differ. Equ. 2015, Article ID 317 (2015)

\section{Submit your manuscript to a SpringerOpen ${ }^{\circ}$ journal and benefit from:}

- Convenient online submission

- Rigorous peer review

- Open access: articles freely available online

- High visibility within the field

- Retaining the copyright to your article

Submit your next manuscript at $\gg$ springeropen.com 